\title{
Anomalias congênitas: fatores associados à idade materna em município sul brasileiro, 2000 a 2007
}

\author{
Congenital anomalies: factors associated with maternal age in municipality \\ south Brazil, from 2000 to 2007
}

\section{Anomalías congénitas: los factores asociados a la edad materna en una ciudad del sur brasileño, 2000 a 2007}

\author{
Willian Augusto de Melo', Robsmeire Calvo Melo Zurita"', Taqueco Teruya Uchimurall', Sonia Silva Marcon ${ }^{\text {IV }}$ \\ 1 Enfermeiro. Mestrando do Programa de Pós-Graduação em Enfermagem (PSE) da Universidade Estadual de Maringá (UEM). Bolsista da \\ Fundação Araucária. Maringá, PR. E-mail: profewill@yahoo.com.br. \\ "Enfermeira. Mestranda do PSE/UEM. Auditora Secretaria Municipal de Saúde de Maringá. Maringá, PR. E-mail: robszurita@bol.com.br. \\ II' Enfermeira. Doutora em Saúde Pública. Docente do PSE/UEM. Maringá, PR. E-mail: taqueco@gmail.com. \\ IV Enfermeira. Doutora em Filosofia da Enfermagem. Docente do PSE/UEM. Maringá, PR. E-mail: soniasilva.marcon@gmail.com.
}

\section{RESUMO}

As anomalias congênitas (AC) constituem importante causa de morbimortalidade infantil. Objetivou-se caracterizar os portadores de AC residentes no município de Maringá no período de 2000 a 2007 relacionando com a idade materna. Os dados foram coletados do Sistema de Informação dos Nascidos Vivos (SINASC) e do Sistema de Informação de Mortalidade (SIM). Do total de nascimentos, $246(0,8 \%)$ eram de AC sendo $100(41 \%)$ portadores de anomalias do sistema músculoesquelético, $39(16 \%)$ do sistema nervoso central, $33(13 \%)$ de fendas palatinas, 23 casos (9\%) de anomalias do sistema urogenital e 51 casos (21\%) para as anomalias cromossômicas, digestivas, cardiovasculares, cabeça e pescoço. A idade materna foi utilizada como variável desfecho na tentativa de explicar a ocorrência da $\mathrm{AC}$ e as variáveis que apresentaram significância estatística em relação à idade materna foram: tipo de gravidez, estado civil, sexo masculino e Apgar no 50 minuto. Com média de 34 óbitos por ano, $70 \%$ dos casos ocorreram em crianças menores de um ano sendo a causa mais frequente o sistema cardiovascular $(40 \%)$. Estudos como este fornecem instrumentos para racionalização da alocação de recursos com base em estratégias estruturadas em informações fidedignas acerca do problema.

Descritores: Anomalias congênitas; Nascimento vivo; Mortalidade na infância; Enfermagem Pediátrica.

\section{ABSTRACT}

Congenital anomalies (CA) constititute important cause of child morbimortality. It was objectified to characterize CA carries residing in the municipality of Maringá in the period between 2000 and 2007 associanting with maternal age. Data were collected from the Information System on Live Births (SINASC) and the Information System on Mortality (SIM). Of the total of births, $246(0.8 \%)$, being $100(41 \%)$ had anomalies of the muscle-skeletal system, $39(16 \%)$ of the central nervous system, $33(13 \%)$ had a cleft palate, 23 cases $(9 \%)$ of anomalies of the urogenital system and 51 cases $(21 \%)$ for the chromosomic, digestive, cardiovascular anomalies, head and neck. The mother's age was used as outcome variable in the attempt to explain the occurrence of the CA and the variable that had presented statistical significance in relation to the mother's age were: multiple births, marital status, male newborn and Apgar $<7$ at the fifth minute. With average 34 deaths per year, $70 \%$ of cases occurred in children under one year being the most frequent cause cardiovascular system (40\%).There was an average of 34 deaths per year, and children less than one year old represented $70 \%$ of cases. The most frequent causes of death from CA were in the cardiovascular system (40\%) and central nervous system (12\%). Such studies contribute to rationalizing the allocation of resources bases on strategies structured on legitimate information.

Descriptors: Congenital abnormalities; Live births; Child mortality; Pediatric Nursing.

\section{RESUMEN}

Las anomalías congénitas (AC) constituyen una causa importante del morbimortalidad infantil. Eso estudio tuvo como objetivo caracterizar el perfil de los portadores de AC residentes en el municipio de Maringá en el período de 2000 a 2007 asociando con la edad materna. Los datos fueron recogidos del Sistema de Información de los Nacidos Vivos (SINASC) y del Sistema de Información de Mortalidad (SIM). Del total de nacimientos, $246(0.8 \%)$ eran de AC siendo 100 (41\%) portadores de anomalías del sistema músculo-esquelético, 39 (16\%) del sistema nervioso central, 33 (13\%) de fisuras palatinas, 23 casos (9\%) de anomalías del sistema urogenital y 51 casos (21\%) del anomalías cromosómicas, digestivas, cardiovasculares, cabeza y cuello. La edad materna fue utilizada pues había sido el variable resultado en la tentativa de explicar la ocurrencia de la AC y de las variables que presentaron significación estadística en relación a la edad materna fueron: tipo del gestación, estado civil, sexo masculinos y Apgar en 5 minuto. Con el promedio de 34 muertes por el año, 70\% de los casos habían ocurrido en niños menores de un año con la causa más frecuente el sistema cardiovascular (40\%). Estos estudios contribuyen para proporcionar instrumentos para racionalización de la ubicación de recursos con base en estrategias estructuradas en informaciones fidedignas acerca del problema.

Descriptores: Anomalías congénitas; Nacimiento vivo; Mortalidad en la infancia; Enfermería Pediátrica. 


\section{NTRODUÇÃO}

Anomalia congênita $(A C)$ é todo defeito funcional ou estrutural, presente no momento do nascimento ou que se manifesta em etapas mais avançadas da vida. Qualquer alteração no decorrer do desenvolvimento embrionário pode resultar em anomalias congênitas que podem variar desde pequenas assimetrias até defeitos com maiores comprometimentos estéticos e funcionais ${ }^{(1)}$. As causas estão ligadas a eventos que precedem ao nascimento, podendo ser herdadas ou adquiridas. Os principais fatores etiológicos são representados pelas condições hereditárias (genéticas), exposição a substâncias (medicamentos, álcool e drogas ilícitas), infecções (citomegalovirose, rubéola e toxoplasmose) e radiações, sendo na maioria das vezes por razões desconhecidas $^{(2)}$.

A incidência de AC encontra-se entre $2 \%$ a $3 \%$ dos nascidos vivos e podem estar localizadas em diferentes órgãos e sistemas. Todas as populações estão expostas aos riscos de desenvolverem malformações congênitas, entretanto, a frequência e o tipo destas malformações variam com a raça, a etnia e as condições socioeconômicas assim como, o acesso aos serviços de saúde, a nutrição, o estilo de vida e a educação materna particularmente às relacionadas ao tubo neural ${ }^{(2)}$. Observa-se alta frequência de malformações menores na raça negra, que são aquelas que não têm implicação funcional ou cosmética importante, como prega simiesca, sindactilia e dedos supranumerários; e de malformações maiores e múltiplas na raça branca, que são as anomalias letais ou que afetam significativamente a função ou a aparência, requerendo tratamento médico ou cirúrgico ${ }^{(2)}$.

Existem métodos preventivos para $70 \%$ dos tipos de AC e para o restante, investimentos em pesquisas são necessários para o conhecimento das causas e mecanismos que levam ao desenvolvimento pré-natal anormal $^{(1)}$. As anomalias congênitas podem ser prevenidas em três níveis. A prevenção primária ocorre principalmente no período pré-concepcional e consiste em evitar a doença, reduzindo a susceptibilidade ou a exposição ao fator de risco. A prevenção secundária é realizada no pré-natal e tem por objetivo evitar a evolução e seqüela da doença através da detecção precoce e tratamento oportuno e a prevenção terciária é realizada no pós-natal tendo por objetivo evitar as complicações da doença através da reabilitação e correção adequadas ${ }^{(1)}$.

A escassez de dados oficiais no Brasil referentes às AC aponta para a necessidade de aprimoramento dos sistemas de informação já existentes, assim como um maior intercâmbio entre os órgãos oficiais do governo e as instituições públicas e privadas com a finalidade de franquear as informações à população ${ }^{(3)}$.
Considerando a mencionada escassez de informações relativas às AC no país, sobretudo, nos aspectos epidemiológicos, sócio-demográficos e estatísticos, este estudo teve como objetivo verificar a associação entre a idade materna dos portadores de AC com as variáveis sócio-demográficas maternas, obstétricas e neonatais, além de caracterizar os óbitos ocorridos por AC.

\section{METODOLOGI A}

Trata-se de um estudo analítico descritivoexploratório, quantitativo, baseado em dados secundários obtidos do Sistema de Informação dos Nascidos Vivos (SINASC) e do Sistema de Informação de Mortalidade (SIM) fornecidos pelo Departamento de Vigilância Epidemiológica da Secretaria Municipal de Saúde de Maringá-PR. A população foi constituída por todos os recém-nascidos e portadores de AC, residentes no Município de Maringá e nascidos no período de 2000 a 2007, assim como todos os óbitos por AC ocorridos no mesmo período.

A partir de 1999, o Ministério da Saúde introduziu um campo específico (campo 34) para o registro das $A C$ na Declaração de Nascidos Vivos e seu código corresponde ao Capítulo XVII da Classificação Internacional das Doenças (CID-10) ${ }^{(4)}$.

A variável dependente foi a idade materna e para verificar se constitui risco para AC optou-se pela categorização das mães em dois grupos: mães adolescentes ( $<20$ anos) e mães tardias ( $\geq 35$ anos) considerando como não risco as mães adultas (20 a 34 anos). As variáveis independentes foram: estado civil, escolaridade materna, duração da gestação, tipo de parto, número de consultas de pré-natal, sexo do recém-nascido, escore de Apgar no primeiro e quinto minuto, raça e peso ao nascer.

O estado civil foi categorizado como casada e não casada sendo que para casada incluíram-se as propriamente ditas casadas e com união consensual e não casada incluíram-se as solteiras, viúvas e divorciadas. Quanto à escolaridade materna considerou-se baixa escolaridade menos de oito anos de estudos e boa escolaridade oito ou mais anos de estudo ${ }^{(5-6)}$

A duração da gestação foi categorizada em menor que 37 semanas e igual ou maior que 37 semanas. O tipo de parto como normal ou cesárea. O número de consultas de pré-natal em menos de sete consultas e sete ou mais consultas. O sexo do recémnascido como masculino e feminino. Os índices de Apgar no primeiro e no quinto minuto em menor que sete e igual ou maior que sete. A raça/cor do recémnascido foi categorizada dicotomicamente como branca e não branca sendo que para a não branca incluiu-se preta, amarela, parda e indígena. O peso ao nascer foi classificado em normal, quando igual ou superior a $2.500 \mathrm{~g}$, e em baixo peso, quando inferior 


\section{a $2.500 \mathrm{~g}^{(5-6)}$.}

Os tipos de AC que determinaram a morte dos indivíduos como causa básica foram coletados por meio do SIM e agrupados nos diversos sistemas orgânicos, de acordo com a CID 10, descrevendo-se as anomalias e agrupando-as em quaisquer dos seguintes grupos de causas: sistema nervoso central (CID10 Q00.0 a Q07.9), sistema cardiovascular (CID10 Q20.0 a Q28.9), anomalias do sistema músculo-esquelético (CID10 Q66.0 a Q79.9), sistema urogenital (CID10 Q60.0 a Q64.9), sistema pulmonar (CID10 Q30.0 a Q34.9), fissura lábio-palatina (CID10 Q35.9 a Q37.9), aparelho digestivo (CID10 Q38.2 a Q45.9), anomalias congênitas de ouvido, face e pescoço (CID10 Q15.0 e Q18.8), anomalias cromossômicas (CID10 Q90.0 a Q99.9) e outras anomalias (CID10 Q80.0 a Q89.9).

Todas as variáveis que apresentaram indicação "ignorado" ou que não apresentaram o campo preenchido foram consideradas como não informadas.
Os dados foram tabulados e codificados pelo Programa Excel 2003. Para verificar associação entre as variáveis e realizar análises multivariadas foi utilizado o teste de qui-quadrado pelo Programa Statistica 7.1. O nível de significância estatística foi definido em $5 \%$ com intervalos de confiança de $95 \%$ para cada variável independente analisada.

Para a realização do estudo foi solicitada autorização formal à Secretaria Municipal de Saúde de Maringá para acesso aos dados secundários. O projeto de pesquisa foi aprovado pelo Comitê Permanente de Ética em Pesquisa Envolvendo Seres Humanos da Universidade Estadual de Maringá (UEM) (Parecer no 620/2008).

\section{RESULTADOS E DI SCUSSÃO}

Constatou-se que no período de 2000 a 2007 ocorreram 32.599 nascimentos de mães residentes no município de Maringá, com $246(0,8 \%)$ casos de AC (Tabela 1).

Tabela 1: Distribuição dos nascimentos segundo presença de anomalias congênitas no período de 2000 a 2007. Maringá-PR, 2008.

\begin{tabular}{|c|c|c|c|c|c|c|c|}
\hline \multirow{2}{*}{ Ano } & \multicolumn{2}{|c|}{ Com Anomalia } & \multicolumn{2}{|c|}{ Sem Anomalia } & \multicolumn{2}{|c|}{ Não I nformado } & \multirow{2}{*}{$\begin{array}{c}\text { Total } \\
\mathbf{N}\end{array}$} \\
\hline & $\mathbf{N}$ & $\%$ & $\mathbf{N}$ & $\%$ & $\mathbf{N}$ & $\%$ & \\
\hline 2000 & 46 & 1 & 3858 & 85,4 & $613 *$ & 13,5 & 3904 \\
\hline 2001 & 39 & 0,9 & 3615 & 90,1 & $358 *$ & 8,9 & 3654 \\
\hline 2002 & 36 & 0,9 & 4161 & 99,0 & 6 & 0,1 & 4197 \\
\hline 2003 & 28 & 0,7 & 4029 & 99,2 & 3 & 0,1 & 4057 \\
\hline 2004 & 23 & 0,5 & 4223 & 99,5 & - & - & 4246 \\
\hline 2005 & 18 & 0,4 & 4161 & 99,5 & 2 & 0,1 & 4179 \\
\hline 2006 & 24 & 0,5 & 4164 & 99,5 & - & - & 4188 \\
\hline 2007 & 32 & 0,7 & 4142 & 99,2 & 1 & 0,1 & 4174 \\
\hline Total** & 246 & 0,7 & 32353 & 96,5 & 12 & 22,8 & 32599 \\
\hline Média & 30,7 & 0,7 & 4044 & 96,5 & $122 *$ & 2,8 & 4198 \\
\hline
\end{tabular}

$\mathrm{X}^{2}$ de tendência $=14,149(\mathrm{p}<0,0001)$

Fonte: SINASC, Secretaria Municipal de Saúde de Maringá-PR.

Estes dados apresentam uma tendência decrescente estatisticamente significativa $(p<0,0001)$ da ocorrência do evento conforme observado nos sete anos de estudo (Tabela 1 ). Acrescentando que a AC apresentou uma média de ocorrência de $0,7 \%$, sendo $1,0 \%$ em 2000 e $0,7 \%$ em 2007, uma redução relativa de $30 \%$. A proporção de recém-nascidos com AC no município de Maringá é semelhante à encontrada em outros estudos que apontaram proporção de 0,4 a 1,5\% dos nascidos vivos(6-7).

Verificou-se que a presença ou ausência de AC não foi registrada no campo específico de 983 $(22,8 \%)$ Declarações de Nascidos Vivos (DN). Uma percentagem mais elevada foi encontrada em Porto Alegre, com $60,7 \%$ de sub-registro para as AC na $D N^{(8)}$ e outro estudo realizado no Vale Paraíba Paulista nos anos de 2002 e 2003 encontrou 8\% de sub-registro ${ }^{(6)}$. Esta ausência de registro praticamente só ocorreu nos dois primeiros anos após a introdução, pelo Ministério da Saúde, do campo 34 sobre "Malformação Congênita e/ou Anomalia Cromossômica" nas DN, apontando para uma possível causa de sub-registros a não familiarização dos profissionais com este campo ${ }^{(9)}$.

Em relação à frequencia dos diferentes tipos de AC, observou-se que 100 recém-nascidos (41\%) eram portadores de anomalias no sistema músculoesquelético dos quais, $70 \%$ deste grupo, eram por malformações no pé ou "pé torto", os demais corresponderam aos casos de malformações de membros superiores, tórax e diafragma. Em seguida, foram encontrados 39 recém-nascidos (16\%) com anomalias do sistema nervoso central que incluíram anencefalia, hidrocefalia, espinha bífida e outras malformações congêneres.

As anomalias craniofaciais incluindo fendas 
palatinas ou fissura lábio-palatal somaram 33 casos $(13 \%)$, as anomalias do sistema urogenital 23 casos (9\%) e as anomalias cromossômicas, digestivas, cardiovasculares, cabeça e pescoço somaram 51 casos $(21 \%)$.

Estudo realizado em 39 municípios do Vale do Paraíba Paulista observou resultados semelhantes nas causas por $\mathrm{AC}^{(6)}$. Outro estudo em 34 maternidades do Município do Rio de Janeiro aponta maiores frequências para anomalias do sistema músculo-esquelético, sistema nervoso central, fenda palatina e sistema geniturinário respectivamente ${ }^{(3)}$.

Quanto a distribuição dos casos de AC por grupo etário materno $65(26,4 \%)$ das mães eram adolescentes, $148(60,2 \%)$ adultas e $33(13,4 \%)$ tardias. Com relação à idade materna um estudo multicêntrico europeu demonstrou maior ocorrência de AC decorrentes de gestações em mulheres com idade superior a 35 anos sendo que as anomalias cromossômicas são mais freqüentes ${ }^{(10)}$. Em contrapartida estudos realizados no Brasil não apontaram associação entre idade materna e anomalia congênita ${ }^{(5,8)}$.

$\mathrm{Na}$ tentativa de explicar a associação entre anomalia congênita e a idade materna a análise univariada das variáveis maternas e da criança apontou significância com o estado civil, escolaridade da mãe, tipo de gravidez e número de consultas prénatal (Tabela 2).

Tabela 2: Análise univariada das variáveis maternas, obstétricas e do recém-nascido segundo a idade materna no período de 2000 a 2007. Maringá, PR, 2008.

\begin{tabular}{|c|c|c|c|c|c|c|c|c|}
\hline \multirow{3}{*}{ Variáveis $(n=246)$} & \multicolumn{6}{|c|}{ I dade Materna } & \multirow{3}{*}{$x^{2 *}$} & \multirow{3}{*}{$\mathrm{p}^{* *}$} \\
\hline & \multicolumn{2}{|c|}{$<20$ anos } & \multicolumn{2}{|c|}{20 a 34 anos } & \multicolumn{2}{|c|}{$\geq 35$ anos } & & \\
\hline & $\mathrm{N}$ & $\%$ & $\mathrm{~N}$ & $\%$ & $\mathrm{~N}$ & $\%$ & & \\
\hline \multicolumn{9}{|l|}{ Estado Civil } \\
\hline Não Casado & 14 & 5,7 & 26 & 10,6 & 20 & 8,1 & \multirow{2}{*}{27,50} & \multirow{2}{*}{$<0,000$} \\
\hline Casado & 51 & 20,7 & 122 & 49,6 & 13 & 5,3 & & \\
\hline \multicolumn{9}{|l|}{ Escolaridade } \\
\hline$<8$ anos & 8 & 3,2 & 36 & 14,6 & 13 & 5,3 & \multirow[b]{2}{*}{9,29} & \multirow{2}{*}{0,0095} \\
\hline$\geq 8$ anos & 57 & 27,2 & 112 & 45,5 & 20 & 8,2 & & \\
\hline \multicolumn{9}{|l|}{ I dade Gestacional } \\
\hline$<37$ semanas & 15 & 6,1 & 38 & 15,5 & 9 & 3,6 & \multirow{2}{*}{0,24} & \multirow{2}{*}{0,8832} \\
\hline$\geq 37$ semanas & 50 & 20,3 & 110 & 44,7 & 24 & 9,8 & & \\
\hline \multicolumn{9}{|c|}{ Tipo Gravidez $(n=207)$} \\
\hline Múltipla & 5 & 2,4 & 2 & 1 & - & - & \multirow{2}{*}{6,34} & \multirow{2}{*}{0,0419} \\
\hline Única & 56 & 27,1 & 115 & 55,6 & 29 & 14 & & \\
\hline \multicolumn{9}{|l|}{ Tipo de Parto } \\
\hline Cesário & 56 & 22,7 & 123 & 50 & 24 & 9,8 & \multirow{2}{*}{2,82} & \multirow{2}{*}{0,2436} \\
\hline Normal & 9 & 3,7 & 25 & 10,1 & 9 & 3,7 & & \\
\hline \multicolumn{9}{|c|}{ № Consultas Pré-natal } \\
\hline$<7$ & 10 & 4,1 & 31 & 12,6 & 13 & 5,3 & \multirow{2}{*}{7,58} & \multirow{2}{*}{0,0225} \\
\hline$\geq 7$ & 55 & 22,3 & 117 & 47,6 & 20 & 8,1 & & \\
\hline \multicolumn{9}{|l|}{ Sexo RN } \\
\hline Masculino & 43 & 17,5 & 92 & 37,4 & 27 & 11 & \multirow{2}{*}{4,63} & \\
\hline Feminino & 22 & 8,9 & 56 & 22,7 & 6 & 2,4 & & 0,0902 \\
\hline Apgar $10 \mathrm{~min}$ & & & & & & & & \\
\hline$<7$ & 15 & 6,1 & 41 & 16,7 & 23 & 9,4 & 0.75 & 06872 \\
\hline$\geq 7$ & 50 & 20,4 & 106 & 43,3 & 10 & 4,1 & 0,13 & $0,08 / 2$ \\
\hline Apgar 50 min & & & & & & & & \\
\hline$<7$ & 8 & 3,3 & 25 & 10,2 & 3 & 1,2 & 174 & 0.4170 \\
\hline$\geq 7$ & 57 & 23,3 & 122 & 49,8 & 30 & 12,2 & 1,14 & $0,41 / 0$ \\
\hline Raça & & & & & & & & \\
\hline Não Branca & 7 & 2,9 & 18 & 7,3 & 4 & 1,6 & 0.88 & 0.9568 \\
\hline Branca & 58 & 23,6 & 130 & 52,8 & 29 & 11,8 & & \\
\hline Peso ao nascer & & & & & & & & \\
\hline$<2500 \mathrm{~g}$ & 10 & 3,7 & 39 & 15,9 & 10 & 4,1 & 494 & 00845 \\
\hline$\geq 2500 \mathrm{~g}$ & 56 & 22,9 & 108 & 44,1 & 23 & 9,4 & 4,94 & 0,0845 \\
\hline
\end{tabular}

$* \mathrm{x}^{2}=$ Qui-quadrado de Pearson.

$* * p=$ Nível descritivo do teste de associação pelo qui-quadrado de Pearson. 
Quanto a idade gestacional $62(25,2 \%)$ dos portadores de AC eram prematuros. Embora não tenha apresentado associação estatística com idade materna, observou-se maior proporção de prematuros entre as mães tardias $(27 \%)$ ao considerar que nove do total de 33 tiveram filhos prematuros (Tabela 2). O índice de prematuridade é considerado alto ao comparar com estudo realizado no município do Rio de Janeiro no período de 1999 a 2001 e outro no Estado de São Paulo no ano de 2001 e 2002 , onde respectivamente encontraram $19 \%$ e $14,9 \%{ }^{(6,9)}$.

O número de cesarianas foi de 203 (82,5\%), sendo que as mães adolescentes foram proporcionalmente submetidas em menor número a este tipo de parto quando comparadas com as mães adultas e as mães tardias (Tabela 2). Embora considerando os diagnósticos intra-útero e a opção médica pelo parto cirúrgico para os casos de AC, estes valores são elevados, pois em outros estudos sobre AC encontraram índices menores de cesarianas como no Vale do Paraíba Paulista $(61,5 \%)$ entre os anos 2002 e 2003 e em Pelotas - RS $(44,4 \%)$ entre os anos 1999 a $2003^{(6,10)}$.

Sobre a realização de consultas pré-natal encontrou-se 54 mães (22\%) com quantidade inadequada de consultas de pré-natal realizadas, apresentando significância estatística na análise univariada $(p=0,0225)$ (Tabela 2$)$. Esta associação pode ser explicada provavelmente porque as mães adolescentes ainda não assumiram a maternidade, ou por acreditar que não há necessidade da assistência mensal através do pré-natal ou mesmo por falta de conhecimento da importância do pré-natal pela idade prematura da mãe. Outro aspecto a ser considerado é a proporção de pré-natais insuficientes entre as mães tardias $(39,4 \%)$ se comparado com os demais grupos de idade materna considerando que 13 do total das 30 mães tardias tiveram número de consultas de pré-natal insuficientes (Tabela 2), fato este preocupante, visto que estas mães, já são adultas, muitas com a vida profissional e afetiva estabilizadas. No Estado de São Paulo encontrou-se quantidades insuficientes de consultas de pré-natal maiores ao comparar com o presente estudo ${ }^{(6)}$.

Em relação ao tipo de gravidez $8(3,4 \%)$ eram de mães com gravidezes múltiplas ressaltando que esta foi a única variável estudada que apresentou 39 $(15,9 \%)$ casos de subnotificações (Tabela 1). Observou-se associação estatisticamente significativa desta variável quando analisada isoladamente com a idade materna $(p=0,0419)$ (Tabela 2$)$ e quando correlacionada com as demais variáveis, a análise multivariada demonstrou risco de 6,14 vezes da mãe adolescente com gravidez múltipla gerar uma criança portadora de AC (Tabela 3). 
Tabela 3: Análise multivariada das variáveis maternas, obstétricas e do recém-nascido em relação ao grupo de mães adolescentes e adultas no período de 2000 a 2007. Maringá-PR, 2008.

\begin{tabular}{|c|c|c|c|}
\hline \multirow{3}{*}{ Variáveis } & \multicolumn{3}{|c|}{ I dade Materna } \\
\hline & \multicolumn{2}{|c|}{$<20$ anos } & 20 a 34 anos \\
\hline & OR* & IC** & p*** \\
\hline \multicolumn{4}{|l|}{ Estado Civil } \\
\hline Não casado & 1,45 & $0,62-3,37$ & 0,3807 \\
\hline \multicolumn{4}{|l|}{ Casado } \\
\hline \multicolumn{4}{|l|}{ Escolaridade } \\
\hline$<7$ anos & 0,50 & $0,20-1,25$ & 0,1439 \\
\hline \multicolumn{4}{|l|}{$\geq 7$ anos } \\
\hline \multicolumn{4}{|l|}{ I dade Gestacional } \\
\hline$<37$ semanas & 1,55 & $0,52-4,60$ & 0,4234 \\
\hline \multicolumn{4}{|l|}{$\geq 37$ semanas } \\
\hline \multicolumn{4}{|l|}{ Tipo Gravidez } \\
\hline Múltipla & 6,14 & $1.01-37,3$ & 0,0484 \\
\hline \multicolumn{4}{|l|}{ Única } \\
\hline \multicolumn{4}{|l|}{ Tipo de Parto } \\
\hline Normal & 0,88 & $0,35-2,23$ & 0,7882 \\
\hline \multicolumn{4}{|l|}{ Cesário } \\
\hline \multicolumn{4}{|l|}{ Sexo RN } \\
\hline Masculino & 2,61 & $0,90-7,54$ & 0,0749 \\
\hline \multicolumn{4}{|l|}{ Feminino } \\
\hline \multicolumn{4}{|l|}{ Apgar $10 \mathrm{~min}$} \\
\hline$<7$ & 1,10 & $0,54-2,25$ & 0,7899 \\
\hline \multicolumn{4}{|l|}{$\geq 7$} \\
\hline \multicolumn{4}{|l|}{ Apgar 5o $\min$} \\
\hline$<7$ & 0,86 & $0,21-3,52$ & 0,8446 \\
\hline \multicolumn{4}{|l|}{$\geq 7$} \\
\hline \multicolumn{4}{|l|}{ Raça } \\
\hline Não Branca & 0,96 & $0,30-3,10$ & 0,9515 \\
\hline \multicolumn{4}{|l|}{ Branca } \\
\hline \multicolumn{4}{|l|}{ Peso ao nascer } \\
\hline$<2500 \mathrm{~g}$ & 0,36 & $0,10-1,22$ & 0,1023 \\
\hline$\geq 2500 \mathrm{~g}$ & & & \\
\hline
\end{tabular}

$*$ OR $=$ Odds Ratio

** IC = Intervalo de Confiança (95\%)

$* * * \mathrm{p}=$ Nível descritivo do teste de Wald

Em Caxias do Sul-RS, estudo que analisou as características das gestações gemelares do ano de 1998 a 2007, encontrou 1,1\% deste tipo de gravidez entre os nascidos vivos com AC sem apresentar significância estatística ${ }^{(11)}$.

Em relação a escolaridade verificou-se que 57 mães $(23,1 \%)$ tinham menos de 8 anos de estudo, compatível ao ensino fundamental incompleto; 130 (54\%) o ensino médio completo ou incompleto e 58 $(23 \%)$ o ensino superior completo ou incompleto. Embora a análise univariada tenha apontado associação entre escolaridade e idade materna (Tabela 2) o mesmo não foi confirmado nas análises multivariadas (Tabelas 3 e 4). 
Tabela 4: Análise multivariada das variáveis maternas, obstétricas e do recém-nascido em relação ao grupo de mães tardias e adultas no período de 2000 a 2007. Maringá-PR, 2008.

\begin{tabular}{|c|c|c|c|}
\hline \multirow{3}{*}{ Variáveis } & \multicolumn{3}{|c|}{ I dade Materna } \\
\hline & \multicolumn{2}{|c|}{$\geq 35$ anos } & \multirow[b]{2}{*}{ p*** } \\
\hline & OR* & $I C^{* *}$ & \\
\hline \multicolumn{4}{|l|}{ Estado Civil } \\
\hline Não casado & 11,42 & $3,90-33,44$ & $<0,0001$ \\
\hline \multicolumn{4}{|l|}{ Casado } \\
\hline \multicolumn{4}{|l|}{ Escolaridade } \\
\hline$<7$ anos & 2,53 & $0,81-7,88$ & 0,1077 \\
\hline \multicolumn{4}{|l|}{$\geq 7$ anos } \\
\hline \multicolumn{4}{|l|}{ I dade Gestacional } \\
\hline$<37$ semanas & 0,80 & $0,13-4,91$ & 0,8150 \\
\hline \multicolumn{4}{|l|}{$\geq 37$ semanas } \\
\hline \multicolumn{4}{|l|}{ Tipo Gravidez } \\
\hline Múltipla & - & - & 0,9963 \\
\hline \multicolumn{4}{|l|}{ Única } \\
\hline \multicolumn{4}{|l|}{ Tipo de Parto } \\
\hline Normal & 0,62 & $0,17-2,27$ & 0,4756 \\
\hline \multicolumn{4}{|l|}{ Cesário } \\
\hline \multicolumn{4}{|l|}{ Sexo RN } \\
\hline Masculino & 3,84 & $0,99-14,80$ & 0,0500 \\
\hline \multicolumn{4}{|l|}{ Feminino } \\
\hline \multicolumn{4}{|l|}{ Apgar $10 \mathrm{~min}$} \\
\hline$<7$ & 1,41 & $0,29-6,76$ & 0,6668 \\
\hline \multicolumn{4}{|l|}{$\geq 7$} \\
\hline \multicolumn{4}{|l|}{ Apgar 5o $\min$} \\
\hline$<7$ & 0,05 & $0,00-0,56$ & 0,0144 \\
\hline \multicolumn{4}{|l|}{$\geq 7$} \\
\hline \multicolumn{4}{|l|}{ Raça } \\
\hline Não branca & 0,99 & $0,18-5,25$ & 0,9986 \\
\hline \multicolumn{4}{|l|}{ Branca } \\
\hline \multicolumn{4}{|l|}{ Peso ao nascer } \\
\hline$<2500 \mathrm{~g}$ & 4,00 & $0,73-21,86$ & 0,1090 \\
\hline$\geq 2500 \mathrm{~g}$ & & & \\
\hline
\end{tabular}

$* \mathrm{OR}=$ Odds Ratio

$* * I C=$ Intervalo de Confiança $(95 \%)$

$* * * p=$ Nível descritivo do teste de Wald

A mãe adolescente, em função do tempo não poderia alcançar níveis mais elevados de escolaridade, acrescentando-se que fatores só cio possam estar associados. Não foram encontrados estudos com resultado semelhante.

Estudo realizado em Guaratinquetá-SP em 1998 encontrou forte associação entre baixa escolaridade materna e marcadores obstétricos de risco para a gestante e o RN, incluindo o baixo peso ao nascer, à perimortalidade, neomortalidade e mortalidade infantil, assim como ao aumento do número de partos ${ }^{(12)}$. Outros estudos concluíram que a menor escolaridade materna se apresentou associada à ocorrência de RN de baixo peso e que as mães com menos de oito anos de estudo têm chance 1,5 vezes maior desta ocorrência. Vale ressaltar a influência da escolaridade onde se notou prevalência elevada de mulheres menos instruídas e associadas a um padrão socioeconômico com menor recurso financeiro( ${ }^{(3,12)}$.

Observou-se que 59 (24\%) dos recém-nascidos com AC apresentaram baixo peso ao nascer não verificando significância estatística ao correlacioná-la isoladamente com os grupos etários maternos (Tabela 2) ou conjuntamente com as demais variáveis (Tabelas 3 e 4). Ao contrário deste, outros estudos apontaram associação entre baixo peso ao nascer e a presença de $\mathrm{AC}^{(13-14)}$. Estudo realizado no Estado de São Paulo e Estado do Rio de Janeiro encontraram proporção estatisticamente significativa $(p<0,001)$ entre o baixo peso e AC com $25 \%$ e 
$18,8 \%{ }^{(6,9)}$. Estudo realizado no Chile no período de 1967 a 1999, a prevalência de defeitos do tubo neural verificou tendência maior em mães com filhos vivos de peso inferior a $1.500 \mathrm{~g}$ e em mães menores de 19 anos de idade ${ }^{(15)}$. Fatores genéticos ou ambientais podem ser responsáveis pela presença de malformações congênitas e estas também estariam implicadas na gênese do baixo peso, explicando, assim, a associação entre a presença de malformações congênitas e $o$ baixo peso ao nascer ${ }^{(16)}$

Referente a variável raça/cor, 217 (88,2\%) dos recém-nascidos eram brancos não havendo correlação entre AC com esta variável nos grupos de idades maternos estudados (Tabela $2,3,4$ ) e nem em outros relatados na literatura ${ }^{(3,6,9)}$.

Com relação ao gênero, prevaleceram às ocorrências de AC para o sexo masculino com 162 casos $(65,9 \%)$ para $84(34,1 \%)$ no sexo feminino. Nota-se a ocorrência discrepante entre os sexos se comparado com outros estudos que também constataram prevalência de AC no sexo masculino, especificamente em anomalias do aparelho respiratório, e ósteomusculares, porém com valores de menor discrepância ${ }^{(6-7,17)}$ com exceção de um estudo que verificou especificamente a prevalência do sexo feminino na anomalia de espinha bífida ${ }^{(16)}$. A análise multivariada evidenciou associação estatística entre $A C$ e a variável sexo para o grupo das mães tardias, inferindo que filhos portadores de $A C$ tem 3,84 mais chances de nascer do sexo masculino entre elas (Tabelas 4).

Ao verificar o estado civil das mães observou-se que $186(75,6 \%)$ eram casadas ou viviam em regime de união consensual e $60(24,4 \%)$ solteiras ou sem companheiro. Este estudo corrobora com estudo realizado no ano 2000 no município de Fortaleza-CE observaram relacionamento estável entre as mães adolescentes $^{(5)}$, pois observou-se proporção maior de solteiras entre as mães tardias $(60 \%)$ ao comparar com os demais grupos etários maternos (Tabela 2). A análise multivariada demonstrou que as mães tardias apresentam 11,4 mais chances de gerar um recémnascido com AC comparadas as mães adultas (Tabela 4).

Em relação ao Apgar no 50 minuto, 209 (85,3\%) dos recém-nascidos com AC apresentaram valor $\geq 7$. Ao comparar com trabalhos que verificaram a prevalência de $A C$ realizados nos Estados de São Paulo e Rio de Janeiro foram encontrados resultados semelhantes, com valores entre $91,4 \%$ e $83 \%$ respectivamente ${ }^{(9,6)}$. A análise multivariada demonstrou que o Apgar no 5ㅇ minuto se apresentou como fator de proteção para as mães tardias (Tabela 4). Para este estudo deve-se considerar a proporção encontrada de Apgar $<7$ entre as mães tardias, que foram menores quando comparados com os demais grupos etários maternos, onde das 33 mães tardias apenas três ( $9 \%$ ) apresentaram o Apgar < 7 (Tabela 2). Ressalta-se que as variáveis Apgar no 10 e no 50 minuto são variáveis importantes e indicadoras para as condições de sobrevida sendo identificadas em alguns estudos como associadas a AC e à morbimortalidade infantil ${ }^{(3,6,9)}$.

Em relação à mortalidade por AC, constatou-se que no período de 2000 a 2007 ocorreram 124 óbitos por AC no município de Maringá nas diversas faixas etárias, com uma média de 34 óbitos por ano, sendo a faixa etária de menores de um ano a mais acometida com 88 casos (71\%), seguida das faixas etárias de 20 a 59 anos com 15 casos (13\%), de seis a nove anos com nove casos $(7 \%)$, de um a cinco anos e de 10 a 19 anos com quatro casos cada (3\%), e de 60 anos e mais com três casos (2\%). Estudo realizado em Pernambuco demonstrou uma elevação do coeficiente de mortalidade em menores de 1 ano por AC contrastando com o decréscimo da taxa de mortalidade infantil dos últimos anos ${ }^{(2)}$.

Dentre as causas de óbito por AC mais frequentes encontramos as $A C$ relacionadas ao sistema cardiovascular (40\%) seguida das anomalias relacionadas ao sistema nervoso central (12\%), sistema digestório $(6 \%)$, sistema respiratório $(6 \%)$, síndromes $(6 \%)$ e outras não especificadas (30\%). Um estudo desenvolvido especificamente sobre a mortalidade determinada por AC aponta semelhanças na prevalência de mortalidade segundo a causa de morte (41\% para sistema cardiovascular, $21 \%$ para sistema nervoso central e $10 \%$ para o sistema digestivo) ${ }^{(2)}$

Ao avaliar a distribuição dos óbitos por AC segundo as variáveis maternas e obstétricas observase que as categorias de risco das variáveis escolaridade e idade materna não apresentaram prevalência sobre as categorias de não risco (Tabela 5). 
Tabela 5: Distribuição dos óbitos por anomalia congênita ocorridos em Maringá - PR no período de 2000 a 2007, segundo variáveis maternas e obstétricas.

\begin{tabular}{|c|c|c|}
\hline Variáveis maternas e obstétricas & $\mathbf{N}$ & $\%$ \\
\hline \multicolumn{3}{|l|}{ Escolaridade materna } \\
\hline$<8$ anos* & 36 & 29,0 \\
\hline$\geq 8$ anos & 47 & 37,9 \\
\hline Ignorado & 41 & 33,1 \\
\hline \multicolumn{3}{|l|}{ I dade Gestacional } \\
\hline$<37$ semanas* & 47 & 37,9 \\
\hline$\geq 37$ semanas & 36 & 29,0 \\
\hline Ignorado & 41 & 33,1 \\
\hline \multicolumn{3}{|l|}{ Peso ao nascer } \\
\hline$<2500 g^{*}$ & 45 & 36,3 \\
\hline$\geq 2500 \mathrm{~g}$ & 35 & 28,2 \\
\hline Ignorado & 44 & 35,5 \\
\hline \multicolumn{3}{|l|}{ I dade materna } \\
\hline$<20$ anos* & 17 & 13,7 \\
\hline 20 a 40 anos & 63 & 50,8 \\
\hline 41 anos e mais* & 2 & 1,6 \\
\hline Ignorado & 42 & 33,9 \\
\hline Total & 124 & 100 \\
\hline
\end{tabular}

* Categoria considerada como risco.

Estudo sobre informações de óbitos em Blumenau apontou resultado semelhante para essas mesmas variáveis ${ }^{(18)}$ assim como no Vale do Paraíba em São Paulo(6).

As variáveis idade gestacional e peso ao nascer apontaram prevalência para o risco de morte. Alguns estudos demonstram resultados semelhantes ou até mais elevados ${ }^{(3,11)}$. Estas variáveis são motivos de hospitalizações prolongadas e de elevadas taxas de morbimortalidade ${ }^{(3)}$.

Em relação a sub-registros observou-se a média de 42 casos (34\%). Estudo que investigou a qualidade de informações de óbitos também encontrou subregistros principalmente nas variáveis relacionadas a gestação, escolaridade e peso ao nascer e ressalta que apesar do sub-registro e da insatisfatória qualidade das informações sóciodemográficas dos óbitos infantis, as causas podem ser utilizadas para planejamento em saúde ${ }^{(18)}$.

Os sub-registros encontrados tanto nas fontes de dados sobre mortalidade como de nascimentos, sugere que o preenchimento destas fontes muitas vezes não são realizados por profissionais da saúde, sendo delegados a funcionários administrativos, frequentemente não treinados para estas funções e por isso incapazes de dimensionar a importância destas informações para programação de ações de saúde, resultando em sub-registro das $A C$ nas estatísticas oficiais ${ }^{(19)}$. Estudo realizado no Estado de Goiás ao descrever a população de recém-nascidos vivos a partir dos dados do Sistema de Informações sobre Nascidos Vivos apontou como limitação do estudo por falhas encontradas no banco de dados devido ao mau preenchimento da DN especialmente nas variáveis relacionadas a presença de $A C$, número de nativivos, natimortos e ocupação materna ${ }^{(20)}$.

\section{CONCLUSÕES}

Estes dados permitem concluir que existe uma tendência decrescente estatisticamente significativa $(p<0,0001)$ da ocorrência do evento conforme observado nos sete anos de estudo, e que a AC apresentou uma média de ocorrência de $0,7 \%$, sendo $1,0 \%$ em 2000 e $0,7 \%$ em 2007, uma redução relativa de $30 \%$.

Os resultados encontrados apontam que existe relação entre idade materna e presença de $A C$ no nascimento. Para as mães adolescentes com gestações múltiplas as chances de gerar uma criança com AC é de 6,14 vezes comparadas as adolescentes com gestação única, e para as mães tardias não casadas, as chances são de 11,4 quando comparadas as mães com idade entre 20 a 34 anos. O Apgar no 5o minuto se apresentou como fator de proteção para este grupo etário.

Idade gestacional e peso ao nascer apontaram prevalência para a mortalidade por $A C$ e os índices de sub-registros encontrados apontam necessidade de implementação de ações educativas junto aos profissionais visando reduzir as deficiências de registro.

Considerando a carência de estudos sobre AC, acredita-se que os resultados apresentados pelo presente estudo poderão contribuir na identificação, discussão das ações para prevenção e assistência aos portadores de $A C$ e às suas famílias além de fornecer instrumentos que propiciem a racionalização e 
alocação de recursos com base em estratégias estruturadas em informações fidedignas acerca do problema.

\section{REFERÊNCI AS}

1. Santos RS, Dias, IMV. Refletindo sobre a malformação congênita. Rev Bras Enferm. 2005; 58(5): 592-96.

2. Arruda TAM, Amorin MMR, Souza ASR. Mortalidade determinada por anomalias congênitas em Pernambuco, Brasil, de 1993 a 2003. Rev. Ass. Med. Bras. 2008; 54(2): 122-26

3. Guerra FAR. Avaliação das informações sobre defeitos congênitos no Município do Rio de Janeiro [thesis]. Rio de Janeiro: Instituto Figueira da Fundação Oswaldo Cruz; 2006. 126 p.

4. Organização Mundial da Saúde. Classificação Estatística Internacional de Doenças e Problemas Relacionados à Saúde, Décima Revisão (CID 10). São Paulo: Editora da Universidade de São Paulo; 2000. $1056 \mathrm{p}$.

5. Magalhães MLC, Furtado FM, Nogueira MB. Gestação na adolescência precoce e tardia: há diferença nos riscos obstétricos? Rev. Bras. Ginecol. Obstet. 2006; 28(8): 446-52.

6. Pinto CO, Nascimento LFC. Estudo de prevalência de defeitos congênitos no Vale do Paraíba Paulista. Rev. paul. pediatr. 2007;25(3):233-39.

7. Ferreira GM, Pereira NGB, Paulin PIA, Machado JC, Huber J. Estudo das frequências de anomalias congênitas em recém-nascidos na Santa Casa de Misericórdia de Ribeirão Preto no período de janeiro/2008 a maio/2008. In: Anais do 54응 Congresso Brasileiro de Genética; [CD-ROM]; 2008 set 16-19; Salvador, Brasil. 2008. 306 p.

8. Senesi LG, Tristão EG, Andrade RP, Krajden ML, Oliveira Junior FC, Nascimento DJ. Morbidade e mortalidade neonatais relacionadas à idade materna igual ou superior a 35 anos, segundo a paridade. Rev. Bras. Ginecol. Obstet. 2004;26(6):477-82.

9. Costa CMS, Gama SGN, Leal MC. Congenital malformations in Rio de Janeiro, Brazil: prevalence and associated factors. Cad. Saude Publica. 2006; 22(11): 2423-31.

10. Cunha J, Aerts D, Leite JCL, Castilha EE. Defeitos congênitos em Porto Alegre: uma investigação da qualidade dos dados registrados na Declaração de Nascido Vivo. Rev. bras. epidemiol. 2002; 5(suppl.1). 11. Souza LH, Madi J M, Araújo BF, Zatti H, Madi SRC, Lorencetti $\mathrm{J}$, et al. Características e resultados perinatais das gestações gemelares (1998-2007). Rev. AMRIGS. 2009;53(2): 150-5.

12. Haidar FH, Oliveira UF, Nascimento LFC. Escolaridade materna: correlação com os indicadores obstétricos. Cad Saude Publica. 2001; 17(4) : 1025-29. 13. Garcia H, Salgueiro GA, Moreno J, Artega C, Giraldo A. Frecuencia de anomalías congénitas en el
Instituto Materno Infantil de Bogotá. Biomedica. 2003; 23(1): 161-72.

14. Nascimento LF, Pinto CO, Proença FP, Gotlieb SL. Prevalência de anomalias congênitas em São José dos Campos, SP, em 2001. Rev. paul. pediatr. 2006; 24(1): 47-51.

15. Nazer J, López-Camelo J, Castilla EE. ECLAMC: estudio de 30 años de vigilancia epidemiológica de defectos de tubo neural en Chile y en Latinoamérica. Rev Med Chil .2001; 129(5):531-39.

16. Cunha CJ, Fontana T, Garcias GL, Martino-Roth MG. Fatores genéticos e ambientais associados à espinha bífida. Rev Bras Ginecol Obstet. 2005;27(5): 268-74.

17. Oliveira IM, Opaleye DTC, Santiago Júnior JF, Sousa ATB, Lima RSA, Grangeiro MPF, et al. Seqüestro pulmonar extralobar: análise anatomopatológica de dois casos em natimortos e revisão da literatura. J. Bras. Patol. Med. Lab. 2008; 44(3): 199-203.

18. Santa-Helena ET, Rosa MB. Avaliação da qualidade das informações relativas aos óbitos em menores de um ano em Blumenau, 1998. Rev. Bras. Saude Mater. Infant. 2003; 3(1):75-83.

19. Righetto ALC, Huber J, Machado JC, Melo DG. Anomalias congênitas: validade das informações das declarações de nascido vivo em uma maternidade de Ribeirão Preto, São Paulo. Pediatria (São Paulo). 2008; 30(3): 159-64.

20. Minamisawa R, Barbos, MA, Malagoni L, Andraus LMS. Fatores associados ao baixo peso ao nascer no Estado de Goiás. Rev. Eletr. Enf. [Internet]. 2005 [cited 2009 set 25];6(3):336-49. Available from: http://www.fen.ufg.br/revista/revista6_3/pdf/04_Ori ginal.pdf.

Artigo recebido em 19.04.09.

Aprovado para publicação em 30.11.09.

Artigo publicado em 31.03.10. 